\title{
Philosophiques
}

\section{Précis de L'esprit et le monde}

\section{John H. McDowell}

Volume 36, numéro 1, printemps 2009

L'idéalisme britannique

URI : https://id.erudit.org/iderudit/038016ar

DOI : https://doi.org/10.7202/038016ar

Aller au sommaire du numéro

Éditeur(s)

Société de philosophie du Québec

ISSN

0316-2923 (imprimé)

1492-1391 (numérique)

Découvrir la revue

Citer ce document

McDowell, J. H. (2009). Précis de L'esprit et le monde. Philosophiques, 36(1), 193-194. https://doi.org/10.7202/038016ar d'utilisation que vous pouvez consulter en ligne.

https://apropos.erudit.org/fr/usagers/politique-dutilisation/ 


\title{
Disputatio
}

\section{Précis de L'esprit et le monde*}

\author{
JOHN H. MCDOWELL \\ Université de Pittsburgh
}

Il peut être difficile de trouver une manière d'attribuer à l'expérience perceptuelle un rapport rationnel - par opposition à un rapport qui n'est pas autre chose que causal - aux jugements perceptuels. Certaines des façons de le faire entrent en conflit avec l'attaque lancée par Wilfrid Sellars sur ce que celui-ci appelle le "mythe du donné ». Ceux qui ne trouveront aucun moyen d'attribuer à l'expérience un rôle fondateur dans la connaissance perceptuelle qui permette en même temps d'éviter de tomber dans le mythe seront tentés de suivre Donald Davidson (et Robert Brandom) en décrétant qu'il n'y a qu'une relation causale entre les activités de la conscience sensorielle et les jugements perceptuels. Mais j'insiste que de telles conceptions ne mènent nullement à une compréhension satisfaisante de l'épistémologie du jugement perceptuel. De plus, ces conceptions tendent à laisser planer un mystère sur la question de savoir comment une pensée empirique peut être dirigée vers le monde tel qu'elle doit l'être pour être une pensée en général, qu'il s'agisse ou non d'une connaissance. À mon avis, l'attrait exercé par l'empirisme n'est pas seulement épistémologique, mais il est également transcendantal au sens où on a besoin de l'empirisme pour rendre intelligible le fait que notre pensée a une visée objective.

On peut attribuer à l'expérience un rôle fondateur sans tomber dans le mythe du donné et ainsi nous armer d'un empirisme satisfaisant, apte à servir à la fois des fins épistémologiques et transcendantales, si nous soutenons que les capacités conceptuelles - des capacités qui appartiennent à la faculté de rationalité consciente et critique d'un sujet - sont actualisées dans de tels sujets, non seulement dans l'activité intellectuelle de décider quoi penser, mais aussi dans la réceptivité de la conscience sensorielle. Comprise comme actualisation des capacités conceptuelles dans la conscience sensorielle, l'expérience peut être une ouverture rationnelle à l'état dans lequel se trouvent les choses. Une expérience peut révéler que les choses sont telles ou telles. Lorsqu'un sujet a une expérience qui révèle que les choses sont telles ou telles, cela peut expliquer comment il se peut qu'un jugement, basé sur l'expérience, selon lequel les choses sont telles ou telles soit une connaissance.

De toute évidence, si nous acceptons une telle conception de l'expérience perceptuelle des animaux rationnels, nous devons tenir un propos différent sur la perception des animaux non rationnels.

"John McDowell, L'esprit et le monde, trad. franç. de C. Alsaleh, Paris, Vrin, 2007, 238 p. 
À mon avis, ce qui bloque la voie à une acceptation toute faite de cette conception de l'expérience des animaux rationnels est l'idée que, possédée comme elle l'est également par les animaux rationnels et non rationnels, la sensibilité - la capacité d'avoir une conscience sensorielle - est un bien naturel, l'idée du naturel étant prise ici en opposition aux idées qui ne fonctionnent que dans le contexte d'une idée de rationalité. Soulignant une caractéristique particulière dont il dit qu'elle appartient à la notion de connaissance, Sellars affirme que le discours sur la connaissance installe son sujet dans l'espace logique des raisons. Il suggère également que le discours sur le naturel installe son sujet dans un espace logique distinct, notamment lorsqu'il affirme qu'une tentative visant à réduire les concepts épistémiques à des concepts non épistémiques tomberait dans une erreur semblable à celle du naturalisme en éthique. En fait, cette séparation conceptuelle est centrale dans l'entreprise de Sellars visant à démasquer le donné comme mythe. Mais si nous acceptons cette séparation conceptuelle, il peut sembler inintelligible que des capacités relevant essentiellement d'une faculté à manœuvrer consciemment et de manière critique dans l'espace des raisons puissent aussi informer notre sensibilité - en supposant que la sensibilité soit quelque chose que nous avons simplement comme animaux.

Pour éviter cette difficulté, il nous faut distinguer le sens dans lequel la sensibilité est naturelle (puisqu'elle appartient à la nature des animaux que nous sommes) de l'idée du naturel qui figure dans cette séparation conceptuelle. Je crois que nous pouvons ici nous aider par un rappel de l'intelligibilité évidente d'une idée de seconde nature. L'actualisation de capacités conceptuelles est une idée qui appartient à l'espace logique des raisons. Néanmoins, les capacités conceptuelles font partie de la seconde nature de leurs possesseurs. La sensibilité en général est une capacité naturelle, mais le sens selon lequel notre version de la sensibilité est naturelle n'implique pas que ses opérations ne puissent être informées par des capacités qui relèvent d'une faculté de rationalité consciente et critique. Ce qu'est pour nous la sensibilité se rapproche de notre seconde nature. Même si Sellars a raison de séparer les concepts qui appartienent à l'espace des raisons des concepts qui servent à caractériser ce que nous pourrions décrire comme ce qui est simplement naturel, notre conception des possibilités de compréhension de la sensibilité des animaux rationnels ne devrait pas être régie par un dualisme entre la raison et la nature animale.

La sensibilité n'est qu'un des aspects de la vie des animaux rationnels que nous risquons de mal comprendre si nous acceptons un dualisme entre la raison et la simple nature. Non seulement ma recommandation d'une sorte d'empirisme sert donc les buts épistémologiques et transcendantaux que j'ai mentionnés plus haut, mais elle sert aussi de zone exemplaire de résistance à tout dualisme de cette sorte. 